\title{
The effect of multisensory stimulation on weight gain in premature infants admitted to the intensive care unit: A clinical trial study
}

\author{
Sari Mahdieh', Mozhgan Rahnama1', Fereshteh Ghaljaei², Majid Reza Akbarizadeh³, \\ Mahin Naderifar ${ }^{4}$
}

${ }^{1}$ School of Nursing and Midwifery, Zabol University of Medical Sciences, Zabol, Iran

${ }^{2}$ School of Nursing and Midwifery, Community Nursing Research Center,

Zahedan University of Medical Sciences, Zahedan, Iran

${ }^{3}$ Department of Pediatrics, School of Medicine, Amir Al-Momenin Hospital,

Zabol University of Medical Sciences, Zabol, Iran

${ }^{4}$ Department of Pediatrics, School of Nursing and Midwifery,

Zabol University of Medical Sciences, Zabol, Iran

\begin{abstract}
Introduction. Premature infants undergo a lot of stressful procedures during care and treatment procedure, which may lead to weight changes. Multisensory stimulation is a broad classification of interventions designed to improve the developmental and physiological outcomes of preterm infants admitted to the neonatal intensive care unit (NICU). The aim of this study was to evaluate the effect of multisensory stimuli on weight gain in preterm infants admitted to the NICU.

Materials and methods. This study was a two-group randomized controlled trial (multisensory stimulation and control group). Forty preterm infants admitted to the NICU who met the inclusion criteria were selected. The intervention method was a multisensory stimulation program including auditory stimulation, tactile stimulation, visual stimulation and vestibular stimulation, which were performed by the researcher for 12 min (each stimulation lasting for $3 \mathrm{~min}$ ). The infant nutrition was monitored and recorded during the intervention and the infant's weight was measured after changing diapers every morning for a 7 days. It was conducted in the two groups using a calibrated scale of confirmed validity and reliability. Data were analyzed using SPSS 20 software, paired t-test and independent t-test.

Results. Based on the findings of the present study, an upturn was observed in weight gain of preterm infants as a result of multisensory intervention. This indicates that the intervention improves weight gain in premature infants. Conclusions. Since premature infants are usually hospitalized in the NICU for a long time due to their low weight and poor physical condition, they are mostly cared by nurses. Hence, considering its positive outcomes, besides specialized care, this effective and very low cost method could be used by nurses to promote weight gain and early discharge of preterm infants.
\end{abstract}

Keywords: multisensory stimulus, weight gain, premature infant, NICU

\section{INTRODUCTION}

One of the major health indicators of a country is its infant mortality rate (1). A preterm infant is a live neonate born before 37 full weeks of pregnancy (2). Despite advances in the medical sciences, the rate of preterm births is still on rise (3). According to the latest statistics released by the World
Health Organization, premature infants account for $11.1 \%$ of births (4). Of four million babies born in the United States each year, an average of $5.12 \%$ are premature (5). Iran is also one of the countries with a high prevalence of preterm labor so that premature infants comprise about $10 \%$ of births (6). One of the most common problems in infants is low birth weight, which is a key indicator of 
health in the society $(7,8)$. Weight is one of the main determinants of physical and cerebral growth in infants, especially premature infants. Infants represent a highly vulnerable group of the society, and the lower birth weight makes them even more susceptible to various factors. Thus, birth weight is one of the leading causes of neonatal mortality in the world $(9,10)$. Infants with low birth weight are particularly prone to problems such as sensory-neurological disorders, cerebral palsy, cognitive and speech delays, neuromotor and visual impairments, hearing loss, behavioral, psychosocial, and school dysfunction (11). Most low birth weight infants suffer from multiple disabilities (12), which may persist until school age and beyond (13). Premature infants also have an extremely vulnerable nervous system that undergoes the fastest period of brain development in the extrauterine environment (14). The growth rate of the central nervous system (CNS) is considerably fast in the third trimester of fetal development (15). Given that with premature birth, CNS growth is inevitably sustained outside the uterus and in the NICU, it may be significantly affected by the environment (16). These infants had to be admitted to NICU in the first weeks of months of life due to a plethora of problems related to prematurity, such as breathing, nutritional, body temperature, jaundice, and other disorders (17). Maintaining physiological stability and weight gain are vital for premature infant discharge $(8,9)$. Since the digestion system of premature infants is weak, it must be improved through various interventions. Pharmacological and non-pharmacological interventions can be beneficial for this purpose. However, in pharmacological interventions, nurses are often in charge of administering drugs, and the medicine may have deleterious effects on weak infants (18).

Non-pharmacological methods such as multisensory stimulation, skin-to-skin contact (19), breastfeeding (20) and non-nutritive sucking (21) can be used to avoid the harmful effects of pharmacological methods. These techniques can effectively improve infant responses through the involvement of various tactile, balance-motor, tactile, or olfactory systems $(22,23)$. Among these techniques, multisensory stimulation is a relatively novel intervention that is inspired by the principles of sensory stimulation therapy (7). Since 1960, researchers have proposed different types of multi- sensory stimulation for hospitalized preterm infants in order to simulate the intrauterine environment in the first weeks of life, and therefore maintain and facilitate development of the preterm infant.

Sensory saturation or multisensory stimulation is a non-pharmacological method to prevent pain-induced physiological changes in infants during stressful procedures, which is currently part of the national guidelines for neonatal pain alleviation (7). The stimulation programs include a combination of auditory, motor, and visual stimulation, with reported positive outcomes in both healthy preterm infants and infants suffering from premature complications (24). Research on premature infants has shown that sensory stimulation, both separately and as a multisensory stimulus, produces positive outcomes in the evolutionary and physiological process such as weight gain, stress reduction, decreased heart rate and positive behavioral changes (9). The study of Nasimi et al. (2015) suggested that multisensory stimulation is a safe and reliable technique for weight gain in premature infants, and nurses can employ this effective and low-cost method to attain this goal (7). It has been shown that various multisensory stimulation techniques can help mitigate some of the adverse neurological effects of preterm birth and the adverse consequences associated with long-term hospitalization (10). Given that infants respond to reactions only through behavioral channels, it is necessary to draw on the expertise and clinical skills of the nurse in infant care to understand the infant's behaviors. So far, several evolutionary models for neonatal care have been proposed for nurses. The professional position of nurses should not be constrained to specialized clinical work such as arterial blood sampling intubation, etc., and the evolutionary interventions should be revisited from a different angle (15). Therefore, one of the proposed strategies for weight gain and stabilization of the physiological state of preterm infants is multisensory stimulation program. The aim of this study was to determine the effect of multisensory stimuli on weight gain in preterm infants admitted to the NICU.

\section{MATERIAL AND METHODS}

This randomized controlled trial study was performed on 80 premature infants admitted to the 
NICU of Amir Al-Momenin Hospital in Zabol. After receiving the code of ethics (IR.ZBMU. REC.1398.102), a letter of introduction from the School of Nursing and Midwifery and permission from the officials of Amir Al-Momenin Hospital in Zabol, we studied premature infants admitted to the NICU of Amir Al-Momenin Hospital Zabol city who met the inclusion criteria. The criteria consisted of a gestational age of 34-30 weeks, 1500-3000 g weight, lack of need for mechanical ventilation or oral feeding through gavage, lack of congenital anomalies, jaundice, eye infection, severe respiratory infections, and asphyxia, no history of cardiopulmonary resuscitation, a 5-min Apgar score of above 6, and parental consent. In case of considerable changes in hemodynamic stability, gastroenteritis, CNS injuries, development of genetic or metabolic diseases during the intervention, infant death and unwillingness and withdrawal of the mother from the research, the infants was excluded from the study.

The sample size $(\mathrm{n}=80)$ was calculated from previous studies using the formula for the comparison of means in two groups with a confidence level (CI) of $95 \%$ and a test power of $90 \%$. Initially, the participants were selected using available sampling method and then randomized into two intervention and control groups. The intervention method received a multi-sensory stimulation including auditory stimulation, tactile stimulation, visual stimulation and vestibular stimulation, which was performed by the researcher for 12 min (each stimulation for $3 \mathrm{~min}$ ). Tactile stimulation was applied by massaging the infant's limbs for $3 \mathrm{~min}$ (while the infant was lying on her side, once on her right side and then on the left side). Six moderate-pressure tactile stimulations were applied to the head, shoulders, hands, back and feet by the fingers each for 10 seconds (25). The auditory stimulation involved playing a lullaby song in the incubator for 3 min (a traditional Iranian lullaby sang with a female voice and played by an Mp3 player in the incubator with an intensity of 65 to $75 \mathrm{~dB}$ (23). The visual stimulation was produced by hanging black and white cards inside the incubator for 3 min. The vestibular stimulation was provided by vertical and horizontal shaking of the infant for 3 min (to do so, the baby was moved up and down on the ramp of the incubator and spun once to the left and once to the right to simulate a horizontal movement (13).

Stimulations were provided to calm and alert infants $30 \mathrm{~min}$ before meals for $12 \mathrm{~min}$ over 7 consecutive days along with routine care. The control group received only routine care. During the stimulation, the neonates were monitored for any signs of stress every 10 seconds without interrupting the stimulation. The intervention was halted for 15 seconds and then resumed if one of the stress symptoms emerged. If the stress symptoms recurred three times in a row, the intervention would be terminated in that session. The infant nutrition was monitored and recorded during the intervention and the infant's weight was measured after changing diapers every morning for a 7 days. It was performed in the two groups using a calibrated scale with confirmed validity and reliability. Weight was measured first prior to the intervention and then the day after intervention and continued daily to compare weight changes with the previous day. Data were analyzed using SPSS20 software as well as paired t-test and independent t-test.

\section{RESULTS}

According to the findings, $50 \%(\mathrm{n}=20)$ of infants in the intervention group were female and $50 \%(\mathrm{n}=20)$ were male. In the control group, $47.5 \%$ were female $(n=19)$ and $52.5 \%$ were male (21\%). The mean gestational age was $32.1 \pm 32.07$ weeks in the intervention group and in $32.37 \pm 1.33$ in the control group. According to the independent t-test, there was no statistically significant difference between the two groups in terms of sex, gestational age, age of entering the study and birth weight (Table 1).

The mean weight of infants on the first day pre-intervention was $1872.5 \pm 466.59 \mathrm{gr}$ in the control group, which increased to $1883 \pm 465.28$ gr after intervention. According to the paired t-test, this weight gain was significant in the control group. The mean weight on the first day pre-intervention was $1834.6+367$ in the intervention group, which surged to $1844.75+368.18$ post-intervention.

According to the paired t-test, this weight gain was significant in the intervention group compared to the pre-intervention $(\mathrm{p}<0.001)$. However, based on independent t-test, there was no statistically significant difference between the intervention and 
control groups one day before and after the intervention $(p=0.68)$. Weight changes on the first and second days in the intervention group demonstrated a significant decline $(\mathrm{p}<0.001)$, but in the control group, a non-significant weight gain $(\mathrm{p}=0.07)$. There was no statistically significant difference between the intervention and control groups in terms of weight changes on the first and second days $(p=0.56)$. Weight changes on the second and third, third and fourth, fourth and fifth, and fifth and sixth days in both intervention and control groups took an upturn. The weight gain was significant in the intervention group $(p<0.05)$ and non-significant in the control group $(\mathrm{p}<0.05)$. Weight changes on the sixth and seventh days in the intervention group revealed a significant growth $(\mathrm{p}<0.05)$ but in the control group, weight dropped but this weight loss was not statistically significant (Table 2).

\section{DISCUSSION}

The results demonstrated that the multisensory stimulation intervention had a significant effect on weight gain in premature infants. The study of Majella et al. (2014) on the impact of tactile and auditory stimulation on rapid weight gain in premature infants induced also showed that premature infants receiving tactile and auditory stimulation experienced higher weight gain than premature infants in the control group (27), which is consistent with the findings of the present study. Ghomi et al. (2019) also investigated the effects of sensory stimulation (28). To do so, they explored the effects of oral motor intervention on oral feeding of preterm infants, with their results suggesting that infants in the intervention group began the first oral feeding (with an average of 7.2 days) as well as eight oral feedings per day (with an average of 13.4 days)

TABLE 1. Demographic characteristics of premature infants admitted to the NICU

\begin{tabular}{|c|c|c|c|}
\hline \begin{tabular}{|l} 
Group \\
Variable
\end{tabular} & $\begin{array}{c}\text { Intervention } \\
\text { Standard deviation } \pm \text { mean }\end{array}$ & $\begin{array}{c}\text { Control } \\
\text { Standard deviation } \pm \text { mean }\end{array}$ & Independent t-test results \\
\hline Fetal weight & $8581.52 \pm 366.04$ & $8192.57 \pm 481.38$ & 0.52 \\
\hline Gestational age & $23.23 \pm 1.07$ & $23.73 \pm 1.33$ & 0.85 \\
\hline Age of entering the study & $3.51 \pm 1.52$ & $3.53 \pm 2.06$ & 0.7 \\
\hline 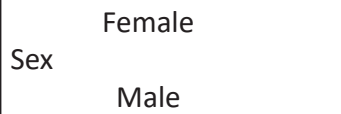 & $\begin{array}{l}20(50) \\
20(50)\end{array}$ & $21(52.5)$ & 0.82 \\
\hline
\end{tabular}

TABLE 2. Comparison of mean weight before and after daily multisensory stimulation intervention in preterm infants admitted to the NICU

\begin{tabular}{|l|c|c|c|}
\hline Group & $\begin{array}{c}\text { Intervention } \\
\text { Weight variable }\end{array}$ & $\begin{array}{c}\text { Control } \\
\text { Standard deviation } \pm \text { mean }\end{array}$ & $\begin{array}{c}\text { Independent t-test results } \\
\text { Standard deviation } \pm \text { mean }\end{array}$ \\
\hline First day & $4,381 \pm 367.65$ & $2,781.05 \pm 466.59$ & 0.68 \\
P & $4,381.57 \pm 368.18$ & $3,881 \pm 465.28$ & 0.68 \\
\hline First day & $<0.005$ & $<0.001$ & 0.68 \\
Secondary day & $43,581.57 \pm 368.18$ & $3,881 \pm 465.28$ & 0.56 \\
P & $9,381.52 \pm 378.30$ & $4,981.05 \pm 467.46$ & 0.56 \\
\hline Second day & $<0.001$ & 0.007 & 0.92 \\
Third day & $9,381.52 \pm 378.30$ & $4,981.05 \pm 467.46$ & 0.92 \\
P & $8,881.52 \pm 424.57$ & $8,981.52 \pm 468.01$ & 0.94 \\
\hline Third day & 0.04 & 0.47 & \\
Fourth day & $8,881.52 \pm 424.57$ & $8,981.52 \pm 468.01$ & 0.94 \\
P & $6,091 \pm 423.84$ & $9,981 \pm 465.34$ & 0.97 \\
\hline Fourth day & 0.001 & 0.92 & \\
Fifth day & $6,091 \pm 423.84$ & $9,981 \pm 465.34$ & 0.97 \\
P & $8,091 \pm 439.66$ & $1,191.05 \pm 470.34$ & 0.05 \\
\hline Fifth day & 0.005 & 0.92 & \\
Sixth day & $8,091 \pm 439.66$ & $1,191.05 \pm 470.34$ & 0.05 \\
P & $6,791.57 \pm 500.82$ & $1,912.73 \pm 1969.29$ & 0.52 \\
\hline Sixth day & 0.01 & 0.48 & \\
Seventh day & $6,791.57 \pm 500.82$ & $1,912.73 \pm 1969.29$ & \\
P & $9,002.52 \pm 516$ & $8,391.05 \pm 465.92$ & 0.43 \\
\hline
\end{tabular}


earlier than the control group, and the length of hospital stay in the intervention group was shorter. In this study, although weight comparison between the two groups did not show a significant difference, the between-group comparisons indicated that weight changes were significant in the intervention group; that is, intervention had brought about a remarkable weight gain (28), which is consistent with the present study. The results of Rangey et al. (2014) study also suggested that sensory stimuli such as massage interventions and kangaroo care effectively improved weight gain and shortened the length of premature infants' stay in the hospital (29). It is aligned with the findings of the current study regarding the effect of sensory stimulation on improving weight gain in preterm infants. The study of Nasimi et al. (2016) on the effectiveness of multisensory stimulation in preterm infants' weight gain demonstrated that the difference between the mean weight on the first day and the discharge day was significantly higher in the intervention group than in the control group. This study recommended multisensory stimulation as a low-cost and effective method to improve premature infant weight gain (25), which is consistent with the present study. In Nasimi's study, multisensory stimulation intervention was provided by the mother of each infant. Considering that the intervention provided by different people may interfere with the effectiveness of the intervention, in the present study, a multisensory stimulation intervention was provided by a trained nurse by controlling possible intervening variables. According to the results, the significant weight gain in the intervention group can be largely due to the multisensory stimulation intervention.

The study of Mohamed et al. (2018) on determining the effect of multisensory stimulation in high-risk infants showed that infants in the multisensory stimulation intervention had a higher weight gain than the control group (26). Fucile and Gisel (2010) studied the effect of sensory-motor interventions on improving motor development and motor function in premature infants (30). In this study, the impact of oral stimulation, tactile motor stimulation and a combined multi-sensory intervention (oral, sensory-motor) on weight gain and motor function of preterm infants was investigated. The results showed that oral, sensorimotor and multisensory stimulation groups experienced more weight gain and improved motor function during the intervention compared to the control group, which is aligned with the present study.

Multisensory stimuli appear to trigger the vagus nerve by stimulating peripheral nerves, which in turn release gastrointestinal hormones such as gastrin and cholecystokinin, thereby facilitating the digestion and absorption of food and weight gain (24). Nadar et al. (2018) study on the effect of physiotherapy intervention versus multisensory stimulation on neurobehavioral growth and weight gain in preterm infants showed that the mean scores of neural development, behavior and weight in the multisensory stimulation group spiked significantly, which is compatible with the present study. The only difference is that in Nadar's study physiotherapy interventions were considerably more effective than multisensory stimulation in improving the coordination of sucking, weight gain, and nerve growth (31). However, the present study only looked into the effects of multisensory stimulation. It seems that stimulating more receptors on the body surface using physiotherapy lays the ground for weight gain in infants (25).

Zeraati et al. (2018) study on the effectiveness of multisensory stimulation in neuromuscular growth in preterm infants revealed that multisensory stimulation can have beneficial effects on neuromuscular growth in preterm infants (32). Fontana et al. (2018) also reported that kangaroo care has positive effects on breastfeeding in preterm infants by promoting maternal self-efficacy and triggering multisensory stimulation, which can accelerate oral feeding (33). Medoff-Cooper et al. (2015) also examined the impact of multisensory stimulation on improving sucking skills in 183 healthy preterm infants who were 24-29 weeks old. The results of this study showed that multisensory stimulation improves feeding behaviors, sucking and oral feeding (34). These studies support the findings of the current study by demonstrating the positive effects of multisensory stimulation intervention. Here, both intervention and control were homogeneous in terms of underlying variables and pre-intervention weight and lacked physical disorders such as congenital anomalies and infection. Also, unlike other studies, the intervention was provided by a trained nurse by controlling all the intervening variables, so that the 
remarkable weight gain in the intervention group could be attributed to the intervention. In light of the positive outcomes of multisensory stimulus on preterm infant weight gain, preterm infant weight gain could be improved by training nurses working in the NICU and regular multisensory stimulation in preterm infants.

One of the limitations of this study was that a host of factors such as environmental noise can influence the hemodynamic status of infants. Thus, we strived to perform interventions under conditions that provided the maximum comfort. On the other hand, following arrangement with NICU officials and staff, we tried to reduce environmental interference factors during the intervention. In addition, since this was a single center trial (samples were taken from the ward of a single hospital) caution should be practiced in generalization of results. Finally, changes related to an intervention may appear over time, but in the present study, like other long-term follow-up studies, we were unable to assess long-term outcomes due to time constraint.

\section{Conflict of interest: none declared \\ Financial support: none declared}

\section{REFERENCES}

1. Zapalo B. Assessing the Effect of a Learning Organization on Change in Levels of Developmentally Supportive Care in the Newborn Intensive Care Unit (Doctoral dissertation, Duquesne University, 2006). Available at: https://dsc.duq.edu/etd/1401.

2. Farhadi R, Yaghobian M, Saravi BM. Clinical findings leading to the diagnosis of sepsis in neonates hospitalized in Imam Khomeini and Bu Ali hospitals, Sari, Iran: 2011-2012. Glob J Health Sci. 2014;6(4):298-303.

3. Verklan MTM, Walden EDS. Core curriculum forneonatal intensive care nursing. 4th ed. WBS Company, 2010.

4. Blencowe H, Cousens S, Chou D, Oestergaard MZ,Say L, Moller A, et al. Chapter 2: 15 million preterm births: Priorities for action based onnational, regional and global estimates. Born TooSoon: The Global Action Report on Preterm Birth New York: March of Dimes, PMNCH, Save theChildren, World Health Organization, 2012.

5. Perlman JM. Neurobehavioral deficits in premature graduates of intensive care - potential medical and neonatal environmental risk factors. Pediatrics. 2001;108(6):1339-48.

6. Behrman RE, Kliegman RM, Jenson HB. The high-risk infant. Textb Pediatr. 1973;451-463.

7. Nasimi F, Behnam Vashani HR, Boskabadi H, Ketabi D. Study the effect of quiet time protocol on physiological characteristics of preterm infants. J Evid Based. 2015;5(1):77-87.

8. Kligeman R. Nelson Textbook of Pediatrics.19th ed. Michigan: Judith Fletcher, 2011;11-59.

9. Lessen BS. Effect of the premature infant oral motor intervention on feeding progression and length of stay in preterm infants. $A d v$ Neonatal Care. 2011;11(2):129-39.

10. Valizadeh L, Asadollahi M. Supports provided by nurses for mothers of premature newborns hospitalized in NICU. Iran J Nurs. 2009;22(28):89-98.

\section{CONCLUSIONS}

Premature infants are usually hospitalized in the NICU for a long time due to their low weight and poor physical condition, and they usually receive routine care by nurses. Therefore, informed by the results of this study about the effectiveness of multisensory stimulation on weight gain in preterm infants, it seems that nurses can, in addition to their specialized care, employ this effective and low-cost technique to promote weight gain and expedite discharge of infants.

\section{Acknowledgements}

This article is based on a master's thesis in Internal-Surgery Nursing and a research project approved by Zabol University of Medical Sciences (code of ethics: IR.ZBMU.REC.1398.102). The authors would like to thank the esteemed Research Deputy of Zabol University of Medical Sciences for supporting this research. Also, we would like to express our gratitude and appreciation to the staff of Amir Al-Momenin Hospital in Zabul, the head nurse and the all nurses of the NICU.

11. Bingham PM, Abassi S, Sivieri E. A pilot study of milk odor on nonnutritive sucking by premature newborns. Arch Pediatr Adolesc Med. 2003;157(1):72-5.

12. Aoyama S, Toshima T, Saito Y, Konishi N, Motoshige K, Ishikawa N, et al. Maternal breast milk odour induces frontal lobe activation in neonates: a NIRS study. Early Hum Dev. 2010;86(9):541-5.

13. Eshgizadeh M, Moshki M, Majeedi Z, Abdollahi M. Modifiable Risk Factors on Preterm Birth: A Case-Control Study. Quarterly of the Horizon of Medical Sciences 2015;21(2):141-6.

14. Standley, Jayne $M$. The effect of music and multimodal stimulation on responses of premature infants in neonatal intensive care. Pediatric Nursing. 2002;24(6):532-8.

15. Fanaroff AA. Obstretic management of prematurity. Martin RJ (ed.). Philadelphia: Mosby, 2010.

16. Gomella TL, Cunningham MD, Eyal FG, Zenk KE. Neonatology: management, procedures, on-call problems, diseases, and drugs. New York; McGraw-Hill, 2004.

17. Han Z, Lutsiv O, Mulla S, McDonald SD. Maternalheight and the risk of preterm birth and low birth weight: a systematic review and meta-analyses. Journal of Obstetrics and Gynaecology Canada. 2012;34(8):721-46.

18. Bellieni CV, Cordelli DM, Marchi S. Sensorial saturation for neonatal analgesia. The Clinical Journal 2007;23(3):219-21.

19. Srivastava S, Gupta A, Bhatnagar A, Dutta S. Effect of very early skin to skin contact on success at breastfeeding and preventing early hypothermia in neonates. Indian J Public Health. 2014; 58:22-6.

20. Harrison D, Reszel J, Bueno M, Sampson M, Shah VS, Taddio A, Larocque $C$, Turner $L$. Breastfeeding for procedural pain in infants beyond the neonatal period. Cochrane Database Syst Rev. 2016 Oct 28;10(10):CD011248. 
21. Golchin M, Sheikhan Soudani E, Bahrami P, Shams Soulari Z. The effect of combined use of nonnutritive sucking and sucrose on cardio-respiratory indices and pain behavior during blood drawingphelebotomy procedures in preterm infants in intensive care unit. J Anesthesiol Pain. 2016;6(4):41-54.

22. Sadat Hossaini A, Negarande R, Mehran A, Movahedi Z. The effect of olfactory stimuli familiar on pain responses following venipuncture in newborns. J Hamedan Univ Med Sci. 2011;18(1):10-9.

23. Goubet N, Strasbaugh K, Chesney J. Familiarity breeds content? soothing effect of a familiar odor on full term newborn. J Dev Behav Pediatr. 2007;28(3):189-94.

24. Symington A, Pinelli J. Developmental care for promoting development and preventing morbidity in preterm infants. Cochrane Database Syst Rev. 2006 Apr 19;(2):CD001814.

25. Nasimi F, Zeraati H, Shahinfar J, Boskabadi H, Ghorbanzade M. The Effect of Multisensory Stimulation on Weight Gain of Preterm Infants. JBUMS. 2016;18(12):13-18.

26. Mohamed EK, Abdelazeim F, Elshafey MA, Nasef N. Neurobehavioral response to multisensory stimulation programme in high-risk neonates. Bulletin of Faculty of Physical Therapy. 2018;23(1):22.

27. Majella M, Santha N, Singh J, Devadason P. Accelerated Weight Gain Among Preterm Infants After Tactile And Auditory Stimulation. Journal of Research in Public Health. 2014;3:1-10.

28. Ghomi H, Yadegari F, Soleimani F, Knoll BL, Noroozi M, Mazouri A. The effects of premature infant oral motor intervention (PIOMI) on oral feeding of preterm infants: A randomized clinical trial.
International Journal of Pediatric Otorhinolaryngology. 2019;120:202-9.

29. Rangey PS, Sheth M. Comparative Effect of Massage Therapy versus Kangaroo Mother Care on Body Weight and Length of Hospital Stay in Low Birth Weight Preterm Infants. International Journal of Pediatrics. 2014;2014:434060.

30. Fucile S, Gisel E. Sensorimotor Interventions Improve Growth and Motor Function in Preterm Infants. Neonatal Network. 2010: (6):359-66.

31. Nadar UD, Thakkar PA, Shah C. Effect of specific physiotherapy interventions in comparison with multisensory stimulation on neurobehavioral outcome and weight gain in preterm infants: a randomized control trial. International Journal of Contemporary Pediatrics. 2018;5(1):178.

32. Zeraati H, Nasimi F, Rezaeian A, Shahinfar J, Zade MG. Effect of Multi-sensory Stimulation on Neuromuscular Development of Premature Infants: A Randomized Clinical Trial. Iranian Journal of Child Neurology. 2018;12(3):32.

33. Fontana C, Menis C, Pesenti N, Passera S, Liotto N, Mosca F, et al. Effects of early intervention on feeding behavior in preterm infants: A randomized controlled trial. Early Human Development. 2018; $121: 15-20$.

34. Medoff-Cooper B, Rankin K, Li Z, Liu L, White-Traut R. Multisensory intervention for preterm infants improves sucking organization. $A d v$ Neonatal Care. 2015 Apr;15(2):142-9. 